\title{
Etude de la Relation entre l'abondance des grands mammifères frugivores et celle des fruits dans le Parc National de Moukalaba-Doudou, Gabon
}

\author{
Fred Loïque MINDONGA NGUELET ${ }^{1,2}$, Christophe Roland ZINGA KOUMBA ${ }^{2 *}$, \\ Jacques François MAVOUNGOU ${ }^{1,2}$, Ephrem NZENGUE ${ }^{1,2}$, Etienne François \\ AKOMO-OKOUE ${ }^{1}$, Yoshihiro NAKASHIMA ${ }^{3}$, Shun HONGO $^{4,5}$, Ghislain Wilfried \\ EBANG ELLA $^{1,2}$, Lilan Brice MANGAMA KOUMBA ${ }^{1,2}$ et Bertrand M'BATCHI ${ }^{1}$ \\ ${ }^{1}$ Université des Sciences et Techniques de Masuku (USTM), BP: 901, Franceville-Gabon. \\ ${ }^{2}$ Institut de Recherche en Écologie Tropicale (IRET-CENAREST), BP: 13354, Libreville-Gabon. \\ ${ }^{3}$ College of Bioresource Science, Nihon University, Fugisawa City, Kanagawa 252-0880, Japan. \\ ${ }^{4}$ Primate Research Institute, Kyoto University Inuyama, Aichi 484-5806, Japan. \\ ${ }^{5}$ Graduate School of Science, Kyoto University, Oiwake-cho, Kitashirakawa, Sakyo-ku, 606-8502 kyoto City, \\ Kyoto, Japan. \\ *Auteur correspondant ; E-mail : ngueletfredloic@yahoo.fr; Tél : +24106 230687.
}

\section{REMERCIEMENTS}

Cette étude a été financée par la JST/JICA, SATREPS.

\section{RESUME}

$\mathrm{Au}$ Gabon, les travaux portant sur les relations plantes animaux demeurent encore très lacunaires. Pourtant, cette connaissance est indispensable dans la compréhension des fonctionnements des écosystèmes forestiers, mais aussi sur le comportement alimentaire des mammifères. C'est dans ce cadre qu'une étude sur l'abondance des mammifères frugivores et celles des fruits a été conduite dans le Parc National de Moukalaba Doudou. Les données sur l'abondance des mammifères ont été recueillies à l'aide des caméras pièges, le long de 11 transects de janvier 2012 à janvier 2014. Quant aux fruits, ils ont été récoltés le long de ces transects sur une bande de $1 \mathrm{~m}$ de large de part et d'autre du transect. Au total, 2492 fruits repartis en 100 espèces ont été recensés au cours de cette étude. Le nombre de fruits recensés a varié en fonction des biotopes prospectés et des mois. Les caméras pièges ont permis d'identifier dix espèces de mammifères frugivores présents dans cette zone. Le test de corrélation $\mathrm{P}$ de Pearson $(\mathrm{R}=0,2 ; \mathrm{df}=10, \mathrm{P}=0,5)$ a montré que la variation entre l'abondance des mammifères et celle des fruits reste indépendante.

(C) 2016 International Formulae Group. All rights reserved.

Mots clés : Abondance, fruits, mammifères, Parc National Moukalaba-Doudou, caméra piège, Gabon.

\section{Relationship between the abundance of frugivores mammals and the fruits in the National Park of Moukalaba-Doudou, Gabon}

\begin{abstract}
In Gabon, the works on animal-plant relationships are still very little known. However, this knowledge is essential in understanding the functioning of forest ecosystems, but also on the feeding behavior of
\end{abstract}


mammals. It is in this context that a study on the abundance of frugivores mammals and those fruits was conducted in the National Park Moukalaba-Doudou. Data on the abundance of mammals were collected using cameras traps along 11 transects from January 2012 to January 2014. As for fruits, they were collected along transects on a $1 \mathrm{~m}$ strip on either side. A total of 2492 fruits divided into 100 species were identified during this study. The number of registered fruit varies by surveyed habitats and months. The camera traps have identified ten species of frugivores mammals in this area. The correlation test $\mathrm{P}$ Pearson $(\mathrm{R}=0.2 ; \mathrm{df}=10, \mathrm{P}=0.5)$ showed that the variation between the abundance of mammals, and fruit remains independent.

(c) 2016 International Formulae Group. All rights reserved.

Keywords: Abundance, Fruits, mammals, National Park of Moukalaba-Doudou, Camera-traps, Gabon.

\section{INTRODUCTION}

Le Gabon, pays d'Afrique centrale, abrite une forêt qui s'étend sur près de $80 \%$ de son territoire (Rieucau, 2001 ; Genini et al., 2009). Ces forêts font parties du Bassin du Congo qui constitue le second plus grand massif forestier tropical après le massif amazonien. Par ailleurs, les forêts du Bassin du Congo représentent l'une des régions du monde les plus riches en espèces animales et végétales (White et Edwards, 2000). En effet, de nombreux travaux scientifiques ont montrés que ces forêts sont caractérisées par des communautés animales très diversifiées et très prisées par les touristes (White et Edwards, 2000). Ces forêts constituent aussi des habitats-clés pour de nombreux mammifères (Rieucau, 2001; Genini et al., 2009). La plupart de ces animaux ont un régime alimentaire de type frugivore. A travers leur comportement alimentaire, ils jouent un rôle important pour la dissémination des graines et la régénération des forêts (White et Edwards, 2000; Haugaasen et Peres, 2007). Aussi, l'analyse des relations plantes-animaux est indispensable dans l'étude du fonctionnement des écosystèmes mais également pour la bonne compréhension de la dynamique des animaux dans les forêts du Bassin du Congo (Théry et al., 1992).

$\mathrm{Au}$ Gabon, les travaux portant sur les relations plante-animaux demeurent encore très lacunaires et fragmentaires. Pourtant, des études antérieures conduites dans plusieurs localités ont mis en évidence les relations entre l'abondance des animaux en particulier frugivores et la production des fruits (Théry et al., 1992; White et Edwards, 2000 ; Vande Weghe, 2012). Par ailleurs, plusieurs études sur l'estimation de la densité des populations de grands mammifères ont été réalisées dans différents sites du pays (White et Edwards, 2000 ; Van Vliet et Nasi, 2007). Cependant, peu d'études ont mis en évidence la corrélation entre l'abondance des fruits et celle des mammifères frugivores. De plus, la connaissance de la dynamique de production des fruits est très importante pour la compréhension de la bioécologie des animaux frugivores. En effet, les fruits fournissent une grande quantité de ressources nutritives pour la plupart des mammifères dans les régions d'Afrique centrale et en particulier au Gabon (Gautier-Hion et al., 1985 ; Hemmons et al., 1983). La production des fruits a une influence sur l'écologie des animaux tels que sur leur régime alimentaire et dans une certaine mesure sur leur reproduction et leur mode d'interaction sociale (Tutin et al., 1997 ; Furuichi et al., 2001). Par exemple, en périodes de rareté des fruits, les mammifères frugivores changent leur composition diététique (White, 1994).

Pour recueillir les informations relatives à la corrélation entre l'abondance des mammifères frugivores et celle des fruits, une étude de nature transversale a été conduite dans le parc national de Moukalaba Doudou au sud-ouest du Gabon. L'objectif de cette étude a été de mettre en évidence l'importance de la production des fruits sur l'abondance des mammifères frugivores afin de comprendre la 
dynamique des populations animales dans cette aire protégée.

\section{MATERIEL ET METHODES}

Site d'étude

L'étude a été menée dans le Parc National de Moukalaba-Doudou (PNMD) situé dans la partie Sud-Ouest du Gabon (Figure 1). Ce parc couvre une superficie de $5028 \mathrm{~km}^{2}$ et est situé à $700 \mathrm{Km}$ de Libreville, capitale politique du Gabon (Iwata et Ando, 2007; Takenoshita et Yamagiwa, 2008; Vande, 2012).

Le climat de cette région est de type équatorial caractérisé par une saison sèche de quatre à cinq mois (mai à septembre) et une saison des pluies de sept à huit mois, allant d'octobre à avril (Takenoshita et al., 2008; Ebang Ella et Yamagiwa, 2014). La pluviométrie annuelle moyenne du parc est de $2000 \mathrm{~mm}$ de précipitations dans l'extrême nord et seulement de $1600 \mathrm{~mm}$ dans le sud. Les températures varient en fonction des saisons. La différence entre les mois les plus frais et les mois les plus chauds (mars et avril) est de l'ordre de 3 à $4{ }^{\circ} \mathrm{C}$. La différence entre la température journalière maximale et minimale augmente avec l'altitude.

Le Parc National de MoukalabaDoudou comprend plusieurs types d'habitats dont les plus importants sont les milieux humides, les savanes, les vieilles forêts secondaires, les forêts de montagnes, les forêts inondables, les jeunes forêts secondaires, les formations rocheuses et les grottes (Vande, 2012, Mounioko et al., 2015, Dibakou et al., 2016). Le milieu anthropisé est constitué par le village Doussala et Mboungou (Iwata et Ando, 2007).

Le paysage floristique de la région comprend de nombreuses espèces rares et endémiques au Gabon (Vande, 2012). Ainsi, la végétation est dominée par plusieurs espèces dont Musanga cecropoides, Aframomum sp, Cissus dinklagie, Myrianthus arboreus, Irvengia gabonensis, Dichostemma glaucescens, Meiocarpidium lepidetum et Diospyros spp. La faune sauvage est représentée par plusieurs espèces de grands mammifères dont les buffles (Syncerus caffer nanus), les éléphants (Loxodonta africana cyclotis), les chimpanzés (Pan troglodytes), les gorilles ( $G$. gorilla gorilla), les hippopotames (Hippopotamus amphibius), les sitatungas (Tragelaphus spekei) et les céphalophes (Cephalophus spp). L'avifaune comprend plusieurs espèces dont l'Euplecte monseigneur (Euplectes hordeaceus) (Lee et al., 2006; Vande, 2012).

La rivière Moukalaba constitue l'un des principaux affluents de la Nyanga. Elle serpente dans une plaine couverte d'une mosaïque de forêts et de savanes.

\section{Collecte des données \\ Recensement des fruits}

Le recensement des fruits a été réalisé sur 11 transects de $2 \mathrm{~km}$ de long répartis comme suit (Figure 2): 2 transects dans les jeunes forêts secondaires; 2 transects dans la forêt marécageuse; 4 transects dans la vieille forêt secondaire; 3 transects dans la forêt de montagnes.

La méthode de collecte utilisée au cours de cette étude a été celle mise au point par Takenoshita et al. (2008). Cette méthode consiste à marcher le long du transect en récoltant tous les fruits (fruits frais, mûrs ou non mûrs, tombés et les restes de fruits mangés par les animaux) sur une bande de 1 $\mathrm{m}$ de large de part et d'autre du transect.

Tous les fruits récoltés ont été ramené au laboratoire, dénombrés puis identifiés jusqu'au niveau de l'espèce à l'aide de la littérature de la flore du Gabon (RapondaWalker et Sillans, 1995).

Tout le recensement des fruits a été effectué de janvier 2012 à janvier 2014 une fois par mois. Par ailleurs, un GPS (Global Positioning System) de marque GARMIN GPS MAP 60 CSX a été utilisé pour marquer l'emplacement des fruits recensés le long des transects.

\section{Recensement des mammifères}

Pour recueillir les informations sur l'abondance des grands mammifères, des cameras pièges (Cam Bushnell Trophy, 2010) 
ont été utilisées. Les sessions de piégeages ont été réalisées en deux étapes.

La première étape a consisté à placer 10 caméras pièges le long de chaque transect à intervalle de $200 \mathrm{~m}$ par piège ; soit un total de 110 caméras pièges sur les 11 transects ; entre janvier 2012 et décembre 2013.

Quant à la deuxième étape, elle a consisté à un déploiement de 5 caméras pièges le long de chaque transect à intervalle de 400 $\mathrm{m}$ par piège, soit un total de 55 caméras pièges, entre décembre 2013 et janvier 2014.

Chaque caméra piège a été attaché à un arbre à une hauteur d'environ $30 \mathrm{~cm}$ du sol et à $10 \mathrm{~m}$ de la ligne du transect (Nakashima, 2015 ; Hongo et al., 2016). Par ailleurs, toutes les caméras pièges ont été géo-référencées dans un GPS de marque GARMIN GPS MAP 60 CSX. Les caméras pièges ont été fixées en « mode vidéo ». Ainsi, des séquences vidéos de 30 secondes de longueur ont été enregistrées. Chaque séquence a été considérée comme la preuve d'une seule visite par une espèce donnée, à condition que l'image ait été obtenue à un intervalle de temps supérieur à 30 minutes après l'image précédente de la même espèce (O'Brien et al., 2003; Nakashima, 2015 ; Hongo et al., 2016). Les cameras-pièges ont été visitées une fois par mois durant la période d'étude afin de changer les batteries et les cartes mémoires SD.

\section{Identification des espèces animales}

Les grands mammifères frugivores ainsi observés ont été identifiés selon les clés de détermination mises au point par Dorst et Dandelot (1993) et Kingdonet al. (2013). Cependant, les rongeurs (petits et grands), carnivores (petits et grands), les ongulés herbivores savanicoles (buffles, cobes, etc.), et les pangolins n'ont pas été pris en compte du fait de leur très petit nombre. Par ailleurs, il a été également difficile de discriminer deux espèces de céphalophes rouges (Cephalophus ogilbyi et Cephalophus callipygus). Ainsi, dans la présente étude, les trois espèces de céphalophes, $C$. dorsalis, $C$. callipygus et $C$. ogilbyi, ont été considéré comme étant
Cephalophus spp. De plus, le statut taxonomique $C$. callipygus et $C$. ogilbyi est très controversée (Lorenzen et al., 2012).

\section{Analyse statistique.}

L'abondance des fruits recensés au sol a été calculée selon la formule suivante: Nombre total des fruits recensés le long des transects/nombre total de mois.

L'abondance des mammifères a été exprimée en taux moyen de capture calculé selon la formule suivante (Tobler et al., 2008 ; Nakashima, 2013b) :

Nombre de vidéos / 1000 jours nuits pièges.

Le test de Pearson (P) a été utilisé pour déterminer la corrélation entre l'abondance des mammifères et celle des fruits.

\section{RESULTATS}

Abondance et diversité des fruits

$\mathrm{Au}$ total, 2492 fruits repartis en 28 familles, 72 genres et 100 espèces ont été récoltés. Par ailleurs, le nombre d'espèces fruitières recensés a varié suivant le biotope prospecté. En effet, le maximum de recensement a été obtenu dans les jeunes et vieilles forêts secondaires, suivi des forêts de montagnes et des marécages (Tableau1).

\section{Abondance et diversité des mammifères frugivores}

$\mathrm{Au}$ total, 80410 nuits-pièges ont été réalisées entre janvier 2012 et Janvier 2014, et 37914 vidéos ont été capturées (événements indépendants ne tenant pas compte des photos réalisées à quelques minutes d'intervalle) durant toute la période d'étude.

Le Parc National de Moukalabadoudou est fréquenté par dix grands mammifères frugivores terrestres et semiterrestres à savoir les chimpanzés (Pan troglodytes troglodytes), les gorilles (Gorilla gorilla), les mandrills (Mandrillus sphinx), cercocèbe à collier blanc (Cerccocebus torquatus torquatus), les éléphants (Loxodonta africana cyclotis), les potamochères (Potamochoerus porcus), les chevrotains aquatiques (Hyemoschus 
aquaticus), les céphalophes bleus (Cephalophus monticola), les céphalophes à dos jaune (Cephalophus sivicultor) et les céphalophes rouges (Cephalophus spp.).

Le Tableau 2 montre que le céphalophe bleu, les céphalophes rouges, le chevrotain aquatique et l'éléphant sont les mammifères les plus abondants dans notre zone d'étude. Les autres espèces notamment le cercocèbe à collier blanc, le céphalophe à dos jaune et le potamochère ont été faiblement représentés. Les grands singes (chimpanzé et gorille) et le mandrill ont présenté un taux de capture $\leq 10$.

\section{Corrélation entre l'abondance des} mammifères et l'abondance des fruits.

La Tableau 3 a montré qu'il y'a une variation de l'abondance des mammifères frugivores et celle des fruits en fonction des mois. En effet, les mammifères frugivores ont été plus abondants de juillet à mars et moins important entre avril et juin. Les fruits ont été plus abondants durant les mois de décembre, de janvier, de février et de novembre. Les plus faibles récoltes de fruits ont été obtenues pendant les autres mois.

Le test de corrélation $\mathrm{P}$ de Pearson $(\mathrm{R}=$ $0,2 ; \mathrm{df}=10, \mathrm{P}=0,5)$ a été positif. Cependant, ce test a demeuré très faible car sa valeur a été proche de zéro. Aussi, ce test a montré que la variation entre l'abondance des mammifères et celle des fruits est resté indépendante (Figure 4).

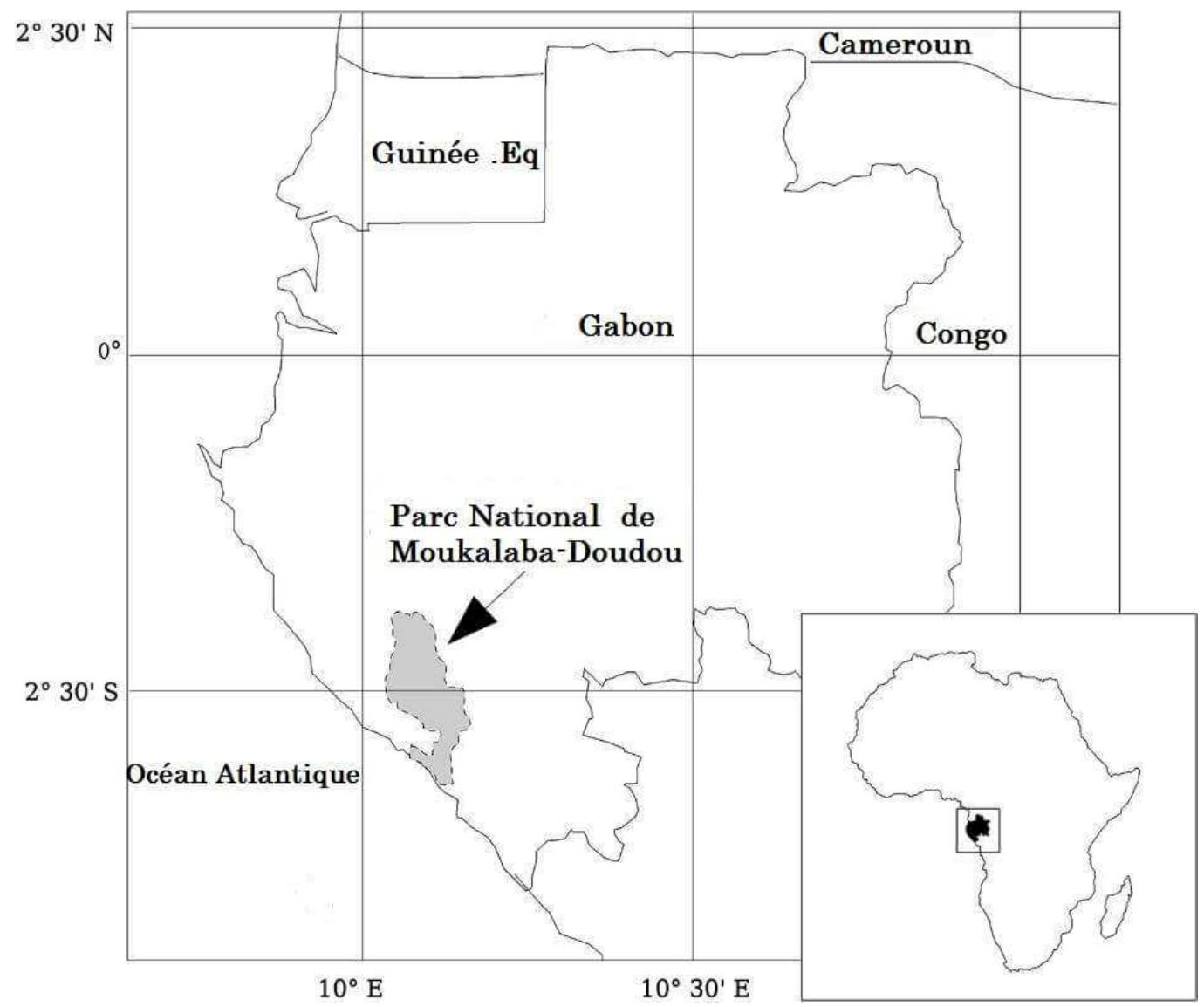

Figure 1 : Localisation du Parc National de Moukalaba-Doudou. 


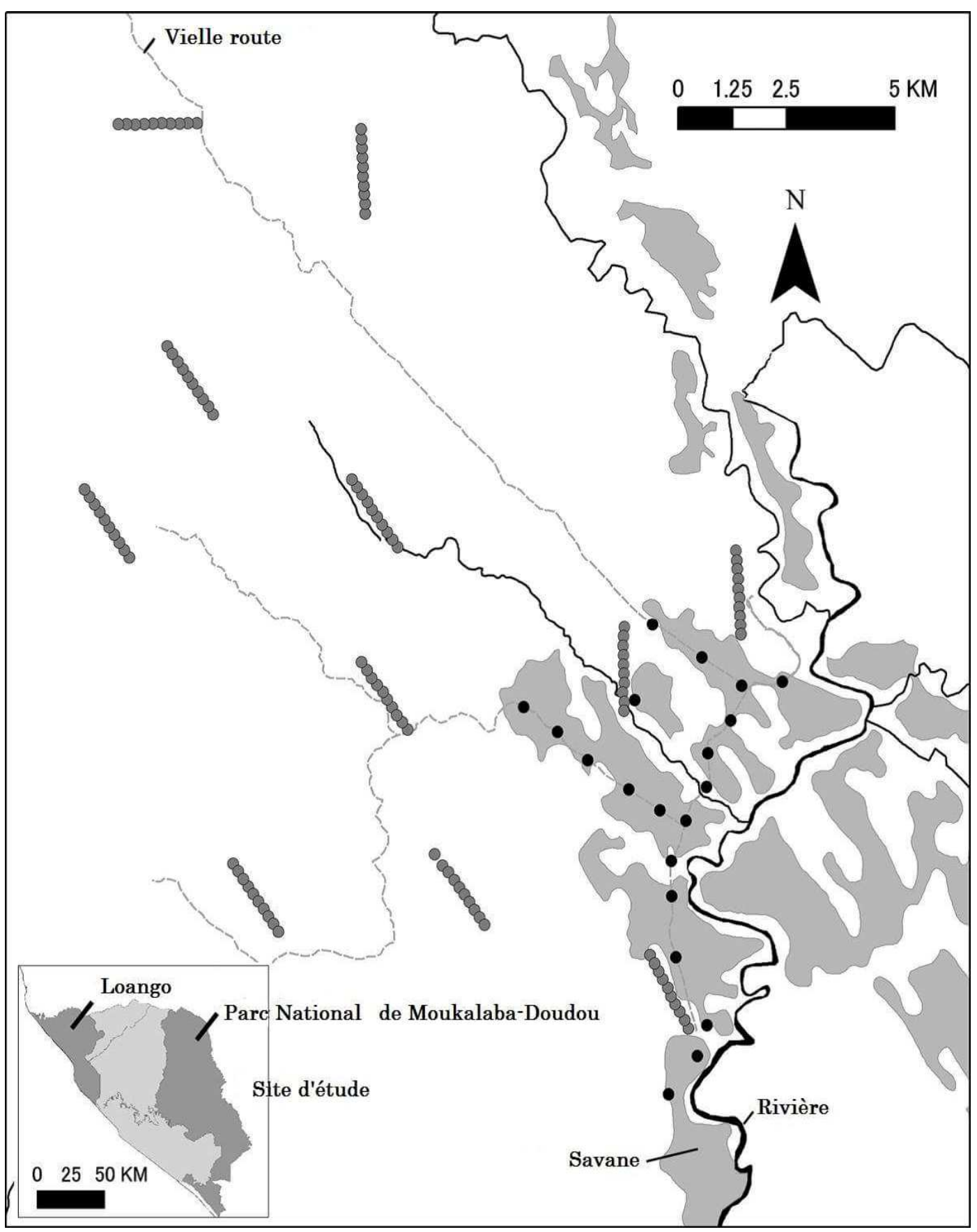

Figure 2: Carte schématique de la zone d'étude dans la partie Est-centrale du parc national de Moukalaba - Doudou, Gabon. Les cercles gris indiquent l'emplacement des caméras pièges dans la forêt, et les cercles noires l'emplacement des caméras pièges dans la savane. Les caméras pièges ont été fixées le long de $2 \mathrm{~km}$ de transects à $200 \mathrm{~m}$ intervalles en forêt. Les barres grises montrent les transects ainsi parcourus. 


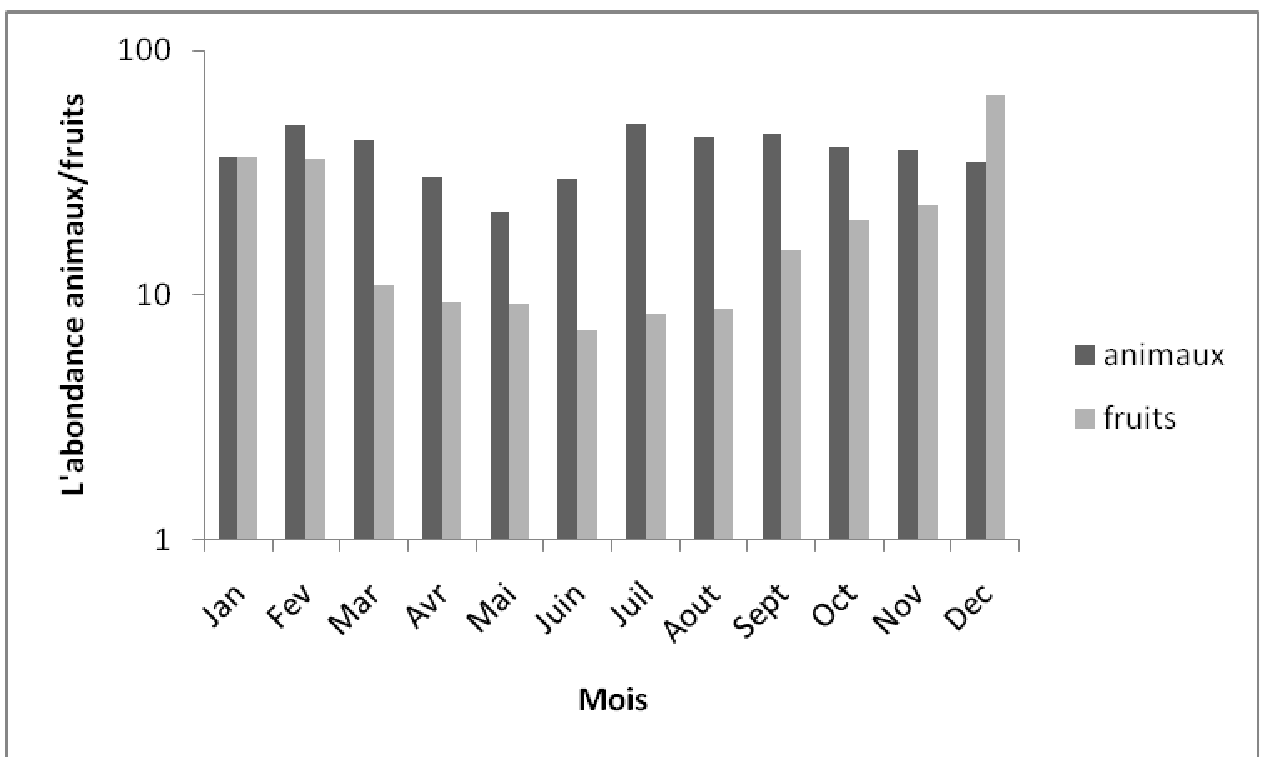

Figure 3: Variation d'abondances des fruits et des grands mammifères en fonction des mois.

Tableau 1 : Liste de famille et des espèces fruitières du Parc National de Moukalaba-Doudou, Gabon.

\begin{tabular}{ll}
\hline Famille & Espèces \\
\hline Annonacea & Annickia chlorantha, Hexalobus sp.1, Meiocarpidium lepidotum, Polyalthia \\
& suaveolens, Uvaria sp.1, Uvariastrum pierreanum, Xylopia quintasii, Xylopia \\
& staudtii, Xylopia hypolampra, Isolona hexaloba, \\
& Pachypodanthium staudtii, Enantia chlorantha, Meiocarpidium lepidotum, \\
& Inconnus \\
\hline Burseraceae & Aucoumea kleineana, Santiria trimera, Canarium schweinfurthii, Inconnus \\
\hline Caesalpiniaceae & Anthonotha fragrans, Aphanocalyx microphyllus, Crudia harmsiana, \\
& Daniellia klainei, Dialum pachyphyllum, Dialum dinklagei, Dialum guineense, \\
& Dialum sp.1, Guibourtiaehie, Guibourtia sp.1, Hylodendrongabunense, \\
& Inconnus \\
\hline Ebanaceae & Diospyros sp.1, Diospyros sp.2, Diospyros sp.3, Diospyros sp.4, Inconnus \\
\hline Sapotaceae & Baillonella toxisperma, Chrysophyllum africanum, Chrysophyllum \\
& lacourtianum, Synsepalum longicuneatum, Tieghemella africana, \\
& Inconnus \\
\hline Mimosaceae & Cylicodiscus gabonensis, Newtoniagriffoniana, Parkia filicoidea, \\
& Piptadeniastrum africanum, Tetrapleura tetraptera, Pentaclethram \\
& acrophylla, Inconnus \\
\hline Euphorbiaceae & Centroplacus glaucinus, Cyrtogononeargentea, Dichostemmaglaucescens, \\
& Drypetesgossweilleri,Drypetes sp.1 Macaranga barteri, Plagiostyles africana, \\
& Ricinodendron heudolotii, Sapium sp.1, Uapaca guineensis, Inconnus \\
\hline Irvingiaceae & Klainedoxa gabonensis, Irvingia grandifolia, Irvingia gabonensis, \\
& Desbordesia sp., Inconnus \\
\hline Myristicaceae & Pycnanthus angolensis, Staudtia gabonensis \\
\hline Meliaceae & Celtistessmannii, Inconnus \\
\hline Entandrophragma utile, Entandrophragma sp.1, Entandrophragma sp.2, \\
Inconnus \\
\hline Craterispermum sp.1, Pausinystalia macroceras, Psydrax sp.1, Inconnus \\
\hline
\end{tabular}


F. L. MINDONGA NGUELET et al. / Int. J. Biol. Chem. Sci. 10(5): 1969-1982, 2016

\begin{tabular}{ll}
\hline Flacourtiaceae & Scottellia klaineana, Scottellia sp.1, Inconnus \\
\hline Simaroubaceae & Quassia sp.1, Inconnus \\
\hline Sapindaceae & $\begin{array}{l}\text { Eriocoelum petiolare, Eriocoelum sp.1, Eriocoelum sp.2, Pancoviasp.1, } \\
\text { Inconnus }\end{array}$ \\
\hline Tiliaceae & Desplatsia sp., Grewiacoriacea, Duboscia macrocarpa, Inconnus \\
\hline Verbenaceae & Vitex doniana, Inconnus \\
\hline Lauraceae & Beilschmiedia sp.1, Beilschmiedia sp.2, Inconnus \\
\hline Lecythidaceae & Petersianthus macrocarpus, Inconnus \\
\hline Zingiberaceae & Aframomum giganteum, Aframomum leptolepis, Inconnus \\
\hline Guttiferae & Garcinia sp.1, Garcinia sp.2, Garcinia sp.3, Inconnus \\
\hline Anacardiaceae & Lannea welwitschii, Pseudospondias longifolia, Trichoscypha abut, \\
& Trichoscypha sp.1, Inconnus \\
\hline Rosaceae & Maranthes glabra, Inconnus \\
\hline Apocynaceae & Funtumia africana, Inconnus \\
\hline Rutaceae & Zanthoxylum gilletii, Inconnus \\
\hline Luxembourgiaceae & Testulea gabonensis, Inconnus \\
\hline Moraceae & Musanga cecropioides, Ficus sp.1, Ficus sp.2, Milicia excelsa, \\
& Myrianthus arboreus, Treculia africana, Inconnus \\
\hline Chrysobalanaceae & Chrysobalanus sp.1, Inconnus \\
\hline
\end{tabular}

Tableau 2 : Liste des grandsmammifères recensés dans le Parc National de Moukalaba-Doudou.

\begin{tabular}{lllc}
\hline Ordre & Nomsscientifiques & Nomscommuns & Taux de capture (n) \\
\hline Primates & Pan troglodytes & Chimpanzé & $9,8(784)$ \\
& Gorilla gorilla & Gorille & $10(804)$ \\
& Mandrillus sphinx & Mandrill & $6,7(538)$ \\
& Cercocebus torquatus & Cercocèbe à collier blanc & $20,9(1678)$ \\
\hline Proboscidea & Loxodonta africana & Elephant & $28,5(2289)$ \\
\hline certartiodactyla & Potamochoerus porcus & Potamochère & $11,9(960)$ \\
& Hyemoschus aquaticus & Chévrotainaquatique & $28,7(2310)$ \\
& Cephalophus monticola & Céphalophe bleu & $201,7(16218)$ \\
& Cephalophus silvicultor & Céphalophe à dos jaune & $18,9(1523)$ \\
& Cephalophus spp & Céphalophes rouges & $134,4(10810)$ \\
\hline
\end{tabular}

n: Nombre global de capture (événements indépendants).

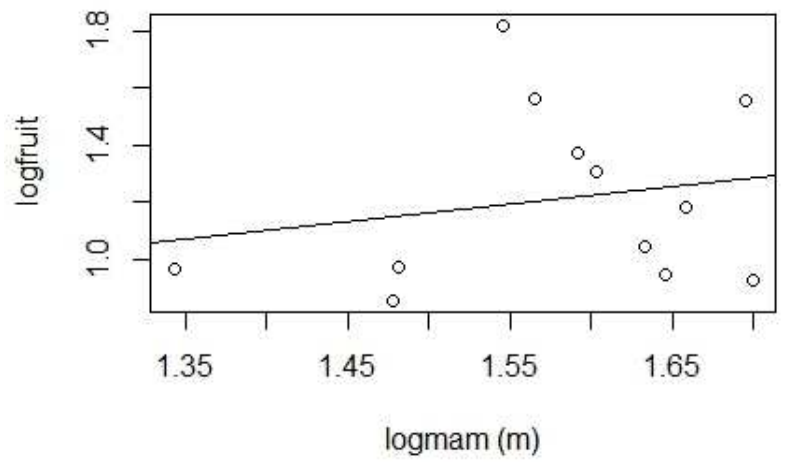

Figure 4: Corrélation entre l'abondance des mammifères frugivores et celle des fruits. 


\section{DISCUSSION}

Les résultats de recensement des fruits obtenus au cours de cette étude ont permis de dresser une liste des familles d'espèces fruitières potentiellement consommées par les animaux en particulier par les mammifères frugivores dans le PNMD. Par ailleurs, la méthodologie utilisée au cours de cette étude a permis de connaitre quelques espèces d'arbres présentes dans la zone d'étude. Aussi, Tutin et White (1998) ont montré que cette méthode donne des résultats comparables aux mesures in situ (sur l'arbre) d'abondance des fruits dans les forêts du Bassin du Congo. Cependant, cette méthode, basée sur le comptage des fruits tombés, présente également des limites. En effet, lorsqu'un fruit est au sol, il peut être retiré du transect par les mammifères frugivores terrestres voire semiterrestres (White et Edwards, 2000).

Par ailleurs, la majorité des grands mammifères vivant dans les forêts tropicales d'Afrique centrale sont frugivores (White et Edwards, 2000). De ce fait, les variations saisonnières de la production des fruits peuvent avoir des implications importantes dans certains comportements chez ces animaux comme le déplacement, la reproduction ou même l'abondance de certains mammifères dans la forêt tropicale (Janzen, 1967). Toutefois, nos résultats suggèrent que; l'augmentation ou la diminution de la production des fruits n'influencerait pas l'abondance des populations des mammifères frugivores terrestres et semi-terrestres. Par conséquent, la production de fruits pourrait ne pas expliquer à elle seule les déplacements ou l'abondance des mammifères frugivores dans cette zone d'étude. Selon une étude conduite par Feer (1988) dans la zone de Makokou, toutes les espèces de céphalophes sont frugivores, mais elles consomment également les jeunes feuilles des arbustes. D'après les travaux de Tutin et al. (1997) menés dans le parc national de la Lopé, les potamochères bien qu'étant frugivores, peuvent se nourrir aussi des racines des plantes herbacées, des Graminées, des graines et même des verres de terre.
Les éléphants et les gorilles sont connus pour avoir une alimentation diversifiée. En effet, les premiers se nourrissent des feuilles, des écorces, des tiges, des racines et de fruits des arbres (White et Edwards, 2000; Remis et al., 2001). Quant aux seconds, en saison sèche; période pendant laquelle les fruits sont rares, ils se nourrissent des feuilles, tiges et des écorces (White et Edwards, 2000). Les forêts (jeunes et vieille forêts secondaires) du PNMD sont riches en Marantaceae et Zingiberaceae, qui sont des sources de nourriture importante pour les éléphants et les gorilles disponibles toute l'année contrairement aux fruits qui sont beaucoup plus disponibles et variés en saison des pluies (Tutin et Fernandez, 1984 ; White, 1994). Par conséquent, la disponibilité des Marantaceae et Zingiberaceae pourrait ainsi expliquer l'abondance de ces animaux durant toute l'année (Tutin et Fernandez, 1984; White, 1994 ; Vliet et Nasi, 2007).

Outre les éléphants et les gorilles qui ont un régime alimentaire diversifié, les chimpanzés, les mandrills et les cercocèbes à collier blanc ont aussi un régime alimentaire assez varié. En effet, le régime alimentaire des chimpanzés se compose principalement des fruits (Tutin et Fernandez, 1993). Cependant, ils peuvent compléter leur régime alimentaire avec des feuilles et des bourgeons de feuilles, des graines, des fleurs, de tiges, de moelle, d'écorce, de résine, des insectes, des oiseaux, des œufs d'oiseau, du miel, de la terre, et des petits mammifères, y compris d'autres primates (Goodall 2001, Boesch et al., 2002). A Moukalaba-Doudou, le mandrill et le cercocèbe à collier blanc sont des espèces qui vivent en sympatrie. Par ailleurs, ils ont pratiquement le même régime alimentaire se nourrissant également des insectes. De ce fait, la diminution de la production en fruit ne semble pas influencer l'abondance de ces animaux.

Selon Dubost (1978), dans la forêt de Makokou, au Nord-Est du Gabon, le régime alimentaire des chevrotains aquatiques est composé presque exclusivement des fruits, mais leur abondance ou densité peut être liée 
au type de végétation. En effet, le chevrotain ne réside que dans les biotopes où se trouvent des abris naturels pouvant leur servir de protection contre les prédateurs (Zinga et al., 2013b; Mindonga-Nguelet et al., 2016). Dans le Parc National de Moukalaba-Doudou, les vieilles forêts secondaires représentent des habitats favorables à ces espèces d'où leur abondance quel que soit le mois dans cette zone d'étude.

Le régime alimentaire des mammifères frugivores semble ne pas être le seul facteur qui pourrait expliquer l'abondance de ces animaux durant toute l'année. Cette abondance pourrait être liée d'une part à la faiblesse de la densité des populations humaines présentes dans la zone d'étude. En effet, la population de Doussala, Mboungou et Konzi (villages périphériques du Parc) qui a été estimée à 143 personnes en janvier 2012 ne chasse pas dans ce parc (Matsuura et Moussavou, 2015). D'autre part, les activités de gestion des aires protégées menées par l'Agence Nationale des Parcs Nationaux semblent avoir un impact sur l'abondance des grands et moyens mammifères présents dans cette zone. En effet, l'interdiction de la chasse contribue à une augmentation de la taille des populations des animaux. Par ailleurs, dans ce parc les animaux sont protégés et par voie de conséquence leur taille ne cesse de croitre.

L'abondance des animaux et la diversité des fruits du PNMD ne montrent pas une différence significative durant les deux saisons. Les résultats obtenus dans cette étude sur la production des fruits est similaire à ceux obtenus dans le parc National de la Lopé (Gabon) et de Ndoki (Congo). En effet, les fruits mûrs sont plus abondants et diversifiés au cours de la saison des pluies à la Lopé (Tutin et al., 1997) et à Ndoki (Kudora et al., 1996). De plus, les facteurs abiotiques tels que la pluviométrie, l'humidité, l'ensoleillement, etc. sont susceptibles d'influencer la maturation des fruits (Takenoshita et Yamagiwa, 2008). White (1994) a répertorié un total de 195 espèces de fruits le long de cinq transects linéaires de $5 \mathrm{~km}$ pendant un an dans le parc national de la Lopé. Takenoshita et Yamagiwa (2008) ont décrit quelques espèces végétales notamment Dispyros spp., Duboscia macrocarpa, Klainedoxa gabonensis, Cissus dinklagei et Sacoglottis gabonensis qui ont une production abondante en fruits durant toute l'année dans le PNMD. Ces espèces végétales sont très prisées par de nombreux mammifères notamment les grands singes et les éléphants. De ce fait, ces espèces végétales joueraient un très grand rôle dans l'abondance des animaux durant toute l'année dans les forêts du Parc National de Moukalaba-Doudou.

\section{Conclusion}

Les fruits constituent une ressource alimentaire fiable pour de nombreuses espèces animales et jouent un rôle vital dans l'écosystème des forêts tropicales. L'étude de la corrélation entre l'abondance des fruits et celle des mammifères frugivores a permis de mettre en évidence une liste d'espèces animales fréquentant le PNMD. Aussi, cette liste pourrait servir de base dans l'étude de la dynamique des populations des mammifères frugivores présents dans cette zone d'étude. Cette étude a aussi permis de mettre en évidence la liste des familles et espèces de fruits potentiellement consommés par les animaux.

Par ailleurs, la méthodologie qui a été utilisée au cours de cette étude pourrait être mise en œuvre dans les autres des aires protégées d'Afrique centrale. De plus, cette méthodologie a présenté l'avantage de prendre en compte l'essentiel des espèces de mammifères frugivores nocturnes et/ou diurnes présentes dans notre site d'étude. En outre, les caméras pièges sont un outil très important pour faire l'inventaire des mammifères terrestres et semi-terrestres présents dans les forêts tropicales d'Afrique. Toutefois cette méthodologie présente des inconvénients dans l'identification de certaines espèces de mammifères frugivores. Aussi, cette méthode doit être combinée avec d'autres techniques pour avoir une liste exhaustive des mammifères frugivores présents dans cette zone d'étude. 
Les résultats obtenus ont montré que l'abondance des mammifères frugivores n'est pas forcement corrélée à celle des fruits. Il existerait d'autres paramètres pouvant avoir une influence sur l'abondance de ces mammifères frugivores.

\section{REMERCIEMENTS}

Nous tenons à remercier l'agence Nationale des Parcs Nationaux (ANPN) et le Centre National de la Recherche Scientifique et Technologique (CENAREST) du Gabon, pour nous permettre de conduire les activités de recherches dans le Parc National de Moukalaba-Doudou. Ce travail a également bénéficié de l'appui institutionnel et logistique de l'Institut de Recherche en Ecologie Tropicale (IRET). Nous remercions aussi J. Yamagiwa, Y. Iwata, C. Ando et les autres membres de PROCOBHA.

\section{REFERENCES}

Amsler S, Filledier J. 1994. Attractivité pour les Tabanidae de l'association métacrésol/octénol. Résultats obtenus au Burkina Faso. Revue d'Élevage et Médecine Vétérinaire des Pays Tropicaux, 47: 93-96.

Barnes RFW, Dunn A. 2002. Estimating forest elephant density in Sapo National Park (Liberia) with a rainfall model. African Journal of Ecology, 40: 159-163.

Boesch C, Boesch-Achermann H. 2000. The Chimpanzees of the Taï Forest: Behavioral Ecology and Evolution. Oxford Univ Pr: Oxford, England; 316 p.

Boesch C, Uehara S, Ihobe H. 2002. Variations in chimpanzee-red colobus interactions. In Behavioral Diversity in Chimpanzees and Bonobos, Boesch C, Hohmann G, Marchant LF (eds). Cambridge Univ Pr: Cambridge, England; 221-30.

Campbell P, Rivera P, Thomas D, BourobouBourobo H, Nzabi T, Alonso A, Dallmeier F. 2006. Structure, composition et diversité floristiques d'une forêt équatoriale du Gabon. Bulletin of the Biological Society of Washington, 12: 29-52.

Dibakou F, Mounioko F, Zinga-Koumba CR, Mbang Nguema OA, Acapovi-Yao GL, Mavoungou JF. 2015. Distribution des Glossines vecteurs de la Trypanosomose humaine africaine dans le Parc National de Moukalaba Doudou (Sud-ouest Gabon). Journal of Applied Biosciences, 86: 7957-7965.

Dorst J, Dandelot P. 1993. Guide des Mammifères d'Afrique. Delachaux et Niestlé : Lausanne.

Dubost G. 1978. Un aperçu sur l'écologie du Chevrotain africain Hyemoschus aquaticus Olgilby, Artiodactyle Tragulidé. Mammalia, 42: 1-62.

Ebang Ella GW, Yamagiwa J. 2014. Use of tool sets by chimpanzees for multiple purposes in Moukalaba-Doudou National Park, Gabon. Primates.

Emmons LH, Gautier-Hion A, Dubost G. 1983. Community structure of the frugivorous-folivorous forest mammals of Gabon. Journal of Zoology Lond, 199: 209-222.

Feer F. 1988. Stratégies écologiques de deux espèces de Bovides sympatriques de la forêt sempervirente africaine (Cephalophus callipygus et C. dorsalis): influence du rythme d'activité. Thèse de Doctorat d'Etat ès Sciences, Université de Pierre et Marie Curie, Paris.

Feer F. 1989. Comparaison des régimes alimentaires de Cephalophus callpygus et C. dorsalis, Bovidés sympatriques de la forêt sempervirente africaine. Mammalia, 53: 563-604.

Furuichi, T, Hashimoto C, Tashiro Y. 2001. Fruit availability and habitat use by chimpanzees in the Kalinzu forest, Uganda: Examination of fallback foods. International Journal of Primatology, 22: 929-945.

Gautier-Hion A, Duplantier JMP, Quris R, Feer F, Sourd C, Decoux JP, Dubost G, Emmons L, Erard C, Hecketsweiler H, Moungazi A, Roussilhon C, Tliiollay JM. 1985. Fruit characters as a basis of fruit 
choise and seed dispersal in a tropical forest vertebrate community. Oecologia, 65: 324-337.

Gautier-Hion A, Michaloud G. 1989. Are figs always keystone resources for tropical frugivorous vertebrates? A test in Gabon. Ecology, 70: 1826-1833.

Genini J, Galetti M, Patricia L, Morellato C. 2009. Fruiting phenology of palms and trees in an Atlantic rainforest land-bridge island. Flora, 204: 131-145.

Goodall J. 2001. Problems faced by wild and captive chimpanzees: finding solutions. In Great apes \& Humans: the Ethics of Coexistence, Beck BB, Stoinski TS, Hutchins M, Maple TL, Norton B, Rowan A, Stevens EF, Arluke A (eds). Smithsonian Inst Pr: Washington DC; p xii-xxiv.

Haugaasen T, Peres CA. 2007. Vertebrate responses to fruit production in Amazonian. Biodivers Conserv, 16: 41654190.

Hongo S, Nakashima Y, Akomo-Okoue ET, Mindonga-Nguelet FL. Female Reproductive Seasonality and Male Influxes in Wild Mandrills (Mandrillus sphinx). International Journal of Primatology.

Iwata Y, Ando C. 2007. Bed and bed-site reuse by western lowland gorillas (Gorilla g. gorilla) in MoukalabaDoudou National Park, Gabon. Primates, 48: 77-80.

Janzen Daniel H. 1967. Synchronization of sexual reproduction of trees within the dry season in Central America. Evolution, 21(3): 620-637.

Kingdon J, Happold D, Butynski T, Hoffmann M, Happold M, Kalina J. 2013. Mammals of Africa (Vol. 1-6). Bloomsbury Publishing: London.

Kuroda S, Nishihara T, Suzuki S, Oko RA. 1996. Sympatric Chimpanzees and Gorillas in the Ndoki Forest, Congo, Mcgrew WC, Merchant LF, Nishida T (eds). Grape Ape Societies, Cambridge University Press: Cambridge ; 71-81.
Lahm SA, Tezi JP. 2006. Etude des communautés de mammifères arboricoles et terrestres de tailles moyennes et grandes à Rabi/Toucan dans le domaine de chasse de Ngové-Ndogo et dans le Sud-Ouest du Parc national de Loango. Bulletin of the Biological Society of Washington, 12: 169-208.

Laws RM, Parker JSC, Johnstone RCB. 1975. Elephants and their Habitats: The Ecology of Elephants in North Bun Yoro, Uganda. Clarendon Press: Oxford; 376p.

Lee ME, Alonso A, Dallmeier F, Campbell P, Pauwels OSG. 2006. The Gamba Complex of Protected Areas: An Illustration of Gabon's Biodiversity. Bulletin of the Biological Society of Washington, 12: 229-242.

Lee PC, Moss CJ. 1995. Structural growth in known-age African elephants (Loxodonta africana). Journal of Zoology, 236: 2941.

Lorenzen E, Heller R, Siegismund HR. 2012. Comparative phylo-geography of African savannah ungulates. Molecular Ecology, 21: 3656-3670.

Matsuura N, Moussavou GM. 2015.Analysis of local livelihoods around MoukalabaDoudou National Park in Gabon. Tropics, 23: 195-2014.

Mindonga Nguelet FL, Zinga Koumba CR, MidokoIponga D, Nzengue E, Mikala Okouyi C, OkouyiOkouyi J, M'batchi B, Mavoungou JF, Mutambwe S. 2016. Identification et caractérisation de la dynamique de la grande faune dans le baï de Momba (nord-est Gabon). International Journal of Biological and Chemical Sciences. (Sous presse).

Mounioko F, Dibakou F, Zinga-Koumba CR, Mbang-Nguema OA, Acapovi-Yao GL, Mutambwe S, et Mavoungou JF. 2015Rythme d'activité journalière de Glossina fuscipes fuscipes, vecteur majeur de la trypanosomiase humaine africaine dans le parc national de Moukalaba Doudou (Sud-ouest Gabon). International Journal of Biological and Chemical Sciences, 9: 419-429. 
Nakashima Y, Iwata Y, Ando C, NzeNkoguee $\mathrm{C}$, Inoue E, Okoue Akomo EF, Mbehang Nguema P, Diop Bineni T, NgokBanak L, Takenoshita Y, Ngomanda A, Yamagiwa J. 2013b. Assessment of Landscape-Scale Distribution of Sympatric Great Apes in African Rainforests: Concurrent Use of Nest and Camera-Trap Surveys. American Journal of Primatology, 75: 1220-1230.

Nakashima Y. 2015. Inventorying mediumand large-sized mammals in the African lowland rainforest using camera trapping. Tropics, 23: 151-164.

Nicolas V, Colyn M. 2003. Seasonal variations in population and community structure of small rodents in a tropical forest of Gabon. Canadian Journal of Zoology, 81: 1034-1046.

O'Brien TG, Kinnaird MF, Wibisono HT. 2003. Crouching tigers, hidden prey: Sumatran tiger and prey populations in a tropical forest landscape. Animal Conservation, 6: 131-139.

Raponda-Walker A, Sillans R. 1995. Les plantes utiles $\mathrm{du}$ Gabon. Essai d'inventaire et de concordance des noms vernaculaires et scientifiques des plantes spontanées et introduites. Description des espèces, propriétés, utilisations économiques, ethnographiques et artistiques. Fondation Raponda-Walker, edition Sépia, centre culturel SaintExupéry (Libreville).

Remis MJ, Dierenfeld ES, Mowry CB, Carroll RW. 2001. Nutritional Aspects of Western Lowland Gorilla (Gorilla gorilla gorilla) Diet Durant seasons of Fruits scarcity at Bai Hokou, Central African Republic. International journal of Primatology, 22: 807-86.

Rieucau J. 2001. Biodiversité et écotourisme dans les pays du centre du golfe de Guinée Prétourisme dans une unité géopolitique instable. Les Cahiers d'Outre-Mer, 216: 417-452.

Takenoshita Y, Ando C, Iwata Y, Yamagiwa J. 2008. Fruit phenology of the great ape habitat in the Moukalaba-Doudou
National Park, Gabon. African Study Monographs Supplementary Issue, 39: 23-39.

Takenoshita Y, Yamagiwa J. 2008. Estimating gorilla abundance by dung count in the northern part of Moukalaba-Doudou National Park, Gabon.African Study Monographs Supplementary Issue, 39: 41-54.

Théry Marc, Erard C, Sabatier D. 1992. Les fruits dans le régime alimentaire de PENELOPE MARAIL (AVE, cracidae) en forêt Guyanaise: Frugivorie stricte et sélective? Revue d'Ecologie, 47: 383401.

Tobler MW, Carrillo-Percastegui SE, Leite Pitman R, Mares R, Powell G. 2008. An evaluation of camera traps for inventorying large- and medium-sized terrestrial rainforest mammals. Animal Conservation, 11: 169-178.

Turkalo A, Fay JM. 1995. Studying elephants by direct observations: preliminary results from the Dzanga clearing, Central African Republic. Pachyderm, 20: 45-54.

Tutin CEG, Fernandez M. 1984. Nationwide census of gorilla (Gorilla g. gorilla) and chimpanzee (Pan t. troglodytes) populations in Gabon. American Journal of Primatology, 6: 313-336.

Tutin CEG, Fernandez M. 1993. Relationships between minimum temperature and fruit production in some tropical forest trees in Gabon. Journal of Tropical Ecology, 9: 241-248.

Tutin CEG, White L, Mackanga-Missandzou A. 1997. The use by rain forest mammals of natural forest fragments in an equatorial African savanna. Conservation Biology, 11: 1190-1203.

Tutin CEG, White LJT. 1998. Primates, phenology and frugivory: Present, past and future patterns in the Lopé Reserve, Gabon. In Dynamics of populationsand Communities in the Tropics, Newbery DM, Prinset HHT, Brown N (eds). Blackwell Science Ltd: Oxford; 309-338.

Van Vliet N, Nasi R. 2007. Mise en évidence des facteurs du paysage agissant sur la 
répartition de la faune dans une concession forestière. Bois et Forêts des Tropiques, 292: 23-37.

Vande Weghe JP. 2012. Moukalaba-Doudou, Agence National des Parcs Nationaux (ANPN), Libreville, Gabon, 296 p.

White LJT. 1994. Biomass of rain forest mammals in the Lopé Reserve, Gabon. Journal of Animal Ecology, 63: 499-512.

White LJT, Edwards A. 2000. Conservation Research in the African Rain Forests: a Technical Handbook. The Wildlife Conservation Society: New York.

Yamagiwa J, Basabose AK, Kaleme KP, Yumoto T. 2008. Phenology of fruits consumed by a sympatric population of gorillas and chimpanzees in Kahuzibiega National Park, Democratic Republic of Congo. African Study Monographs Supplementary Issue, 39: 3-22.
Zinga Koumba RC, Acapovi Yao GL, Mavoungou JF, Tongue Kohagne TL, Mbang Nguema OA, Obame Ondo KP, Shango M. 2013-. Influence de la saison sur l'écodistribution des glossines, tabanides, stomoxes du Baï de Momba Makokou, Gabon. Agronomie Africaine, 25: 149-158.

Zinga-Koumba RC, Bouyer J, Mavoungou JF, Acapovi Yao GL, Kohagne TL, Mbang Nguema OA, Obame Ondo KP, Mutambwe S. 2013b. Evaluation de la diversité des diptères hématophages dans une clairière marécageuse du Gabon à l'aide des pièges Vavoua et Nzi. Revue d'Elevage et de Médecine Vétérinaire des Pays Tropicaux, 66: 91-96. 